Revue des patrimoines

$42 \mid 2020$

Imagerie numérique et patrimoine culturel :

représentation et transmission des connaissances

\title{
Les enjeux juridiques liés à la numérisation tridimensionnelle du patrimoine
}

Legal issues involved in the 3D digitisation of heritage

\section{Samuel Bonnaud-Le Roux}

\section{OpenEdition}

Journals

Édition électronique

URL : http://journals.openedition.org/insitu/27773

DOI : $10.4000 /$ insitu. 27773

ISSN : 1630-7305

Éditeur

Ministère de la Culture

Référence électronique

Samuel Bonnaud-Le Roux, « Les enjeux juridiques liés à la numérisation tridimensionnelle du

patrimoine », In Situ [En ligne], 42 | 2020, mis en ligne le 29 juin 2020, consulté le 12 janvier 2021. URL :

http://journals.openedition.org/insitu/27773; DOI : https://doi.org/10.4000/insitu.27773

Ce document a été généré automatiquement le 12 janvier 2021.

\section{cc) () $९$}

In Situ Revues des patrimoines est mis à disposition selon les termes de la licence Creative Commons Attribution - Pas d'Utilisation Commerciale - Pas de Modification 4.0 International. 


\title{
Les enjeux juridiques liés à la numérisation tridimensionnelle du patrimoine
}

\author{
Legal issues involved in the 3D digitisation of heritage
}

\author{
Samuel Bonnaud-Le Roux
}

\section{Introduction}

1 La numérisation tridimensionnelle du patrimoine occupe une place grandissante dans les institutions culturelles. Pour les musées, les services d'archives ou les bibliothèques, elle est un levier non seulement pour faire évoluer leurs missions traditionnelles de conservation et de recherche mais aussi pour valoriser le patrimoine à travers les nouvelles pratiques numériques. En termes de politique publique, elle répond à plusieurs objectifs : le rayonnement de la culture française dans le monde, sa visibilité sur Internet, l'accessibilité à tous les citoyens des collections des musées et l'adaptation de ces derniers aux nouveaux usages des publics' ${ }^{1}$. Elle se trouve donc au cœur d'une des principales missions du ministère de la Culture, affirmée dès le premier article du décret qui fixe ses attributions ${ }^{2}$ : «Le ministre de la Culture a pour mission de rendre accessibles au plus grand nombre les œuvres capitales de l'humanité et d'abord de la France ».

2 La présente réflexion portera sur la numérisation d'objets qui ont pu être protégés dans le passé par le droit d'auteur mais dont la durée de protection a atteint son terme : il s'agit d'œuvres situées dans le domaine public ${ }^{3}$. La question qui se pose est de savoir comment s'articule la libre réutilisation de telles œuvres avec leurs reconstitutions numériques, qui sont susceptibles d'être protégées à leur tour par la propriété littéraire et artistique. Lorsqu'une institution culturelle réalise un modèle numérique 3D d'un objet de ses collections, de multiples questions juridiques surgissent concernant le statut des images produites. Quels sont les droits de propriété ou d'usage sur la numérisation, qui les détient et pour quels usages? 
Bien que des travaux soient disponibles sur des sujets connexes ${ }^{4}$, il n'existe pas à ce jour d'étude consacrée à cette question. Après avoir, dans un premier temps, clarifié ce qu'on entend par "domaine public» et "numérisation en trois dimensions du patrimoine ", nous verrons comment se combinent les deux principales facettes du droit concernées par la numérisation: le droit d'auteur d'une part, le droit de la réutilisation des informations publiques d'autre part.

\section{Un travail de définition préalable}

\section{Précision sémantique sur la notion de « domaine public »}

4 L'expression "domaine public », polysémique, prête parfois à confusion. Elle peut désigner en droit deux réalités différentes, selon le sens que prend l'adjectif « public »: au sens du droit applicable à une personne publique ou au sens de commun ou accessible à tous. D'un côté, le domaine public peut désigner les biens physiques appartenant à des personnes publiques, qui sont soit affectés à l'usage direct du public, soit affectés à un service public. Pris en ce sens, le domaine public est régi par le Code général de la propriété des personnes publiques. Mais d'un autre côté, au sens de la propriété intellectuelle, le domaine public renvoie aux œuvres qui ne sont plus protégées par le droit d'auteur. Les collections anciennes des musées, comme les sculptures du département des Antiquités grecques, étrusques et romaines du Louvre, font partie du domaine public à ce double titre.

\section{Des pratiques de numérisation protéiformes}

Définir la numérisation est un premier défi car elle renvoie à des situations très variées. L'Association des professionnels de l'information et de la documentation (ADBS) propose la suivante: "processus de conversion d'un signal analogique en un signal numérique. Cette technique permet de stocker des documents, quels qu'en soient la nature ou le support, sous une forme électronique. » Appliquée au patrimoine, la notion de numérisation renvoie à une palette de techniques permettant de dupliquer un bien culturel pour aboutir à sa représentation numérique en trois dimensions, réalisée à l'aide d'un logiciel informatique de modélisation. Elle se décline aujourd'hui dans les différentes composantes du patrimoine.

Dans le domaine de l'archéologie, en raison des difficultés d'accès et de la fragilité naturelle des grottes, le Centre national de préhistoire et les directions régionales des Affaires culturelles s'orientent vers la réalisation de répliques à l'identique qui sont visitables par le public, dont l'exemple le plus emblématique est Lascaux IV. Le ministère de la Culture a également produit une collection numérique de référence, "Grands sites archéologiques", accessible en ligne ${ }^{5}$.

Dans le secteur muséal, l'Agence photographique de la Réunion des musées nationaux Grand Palais porte le projet "France Collections 3D », qui vise à accroître la diffusion des œuvres majeures du patrimoine français en produisant des modèles 3D d'une partie des collections des musées afin de répondre à plusieurs objectifs comme la mise à disposition de chercheurs d'un substitut à un objet original fragile, le développement des publics ou la réalisation de projets de réalité augmentée et de jeux vidéo ${ }^{6}$. Les numérisations sont publiées en ligne, sur un compte dédié sur la plateforme Sketchfab7 
8 Enfin, la modélisation 3D peut également être un recours pour reconstituer des bâtiments historiques malheureusement détruits par la guerre ou le terrorisme. La start-up française Iconem est un spécialiste mondial en la matière et s'est fait connaître par son travail de photographie puis de reproduction en 3D de la cité antique de Palmyre, détruite par Daech en Syrie. En 2017, le ministère de la Culture a ainsi mis à disposition des photos d'archives inédites qui ont permis à la start-up Iconem, sur la base de ses relevés 3D réalisés par drone sur site, de reconstituer un modèle 3D du temple de Bêl de Palmyre ${ }^{8}$.

\section{La numérisation en 3D au prisme du droit d'auteur}

9 La rencontre entre numérisations du patrimoine et droit d'auteur met au défi le champ d'application de ce dernier: il importe de faire le départ entre celles qu'il protège comme œuvres de l'esprit et celles qui ne sont pas éligibles à sa protection.

Défini par l'article L. 1 du Code du patrimoine comme «l'ensemble des biens, immobiliers ou mobiliers, relevant de la propriété publique ou privée, qui présentent un intérêt historique, artistique, archéologique, esthétique, scientifique ou technique ${ }^{9}$ ", le patrimoine englobe naturellement de nombreux objets qui sont des œuvres de l'esprit au sens du droit d'auteur. En fonction de l'ancienneté de leur auteur, elles sont soit encore protégées, soit entrées dans le domaine public. Ce dernier constitue un fonds commun où chacun est libre de puiser : dans un processus cumulatif qui traverse toute l'histoire de l'art, les créations d'hier nourrissent celles de demain. À cette distinction initiale s'ajoute une troisième hypothèse de rencontre entre patrimoine et droit d'auteur: l'inspiration que puisent des œuvres contemporaines dans des œuvres antérieures.

11 Le droit d'auteur tient compte de cette réalité en protégeant les œuvres composites et les œuvres dérivées, deux notions qui sont considérées comme synonymes par la doctrine et la jurisprudence. Les premières sont définies comme les œuvres nouvelles à laquelle sont incorporées des œuvres préexistantes sans la collaboration de l'auteur de ces dernières ${ }^{10}$. Quant aux secondes, elles rassemblent différents cas de figure, énumérés par la loi : ce sont par exemple les traductions, adaptations, arrangements, ou encore les bases de données qui constituent des créations intellectuelles ${ }^{11}$. Ces catégories d'œuvres sont protégées par le droit d'auteur pourvu qu'elles soient originales.

\section{L’appréciation de l'originalité}

12 Le droit d'auteur protège comme œuvre de l'esprit toute création humaine qui réunit deux conditions : revêtir une forme perceptible par les sens et être originale. À dessein, le législateur n'a pas défini cette notion d'œuvre de l'esprit dans le Code de la propriété intellectuelle (CPI) mais a choisi de ne prévoir qu'une liste indicative des œuvres protégeables $^{12}$. Il s'agit notamment des œuvres littéraires, musicales, plastiques, audiovisuelles, photographiques ou encore des logiciels. La jurisprudence a précisé et complété cette liste au fur et à mesure de l'évolution de l'art et de la technique. La notion d'originalité n'est pas davantage définie afin de ne pas figer des critères qui pourraient rapidement devenir obsolètes. Par conséquent, en cas de litige, la loi laisse aux juges du fond le soin de trancher la question de l'originalité, en s'en remettant à 
leur pouvoir souverain d'appréciation des circonstances de fait qui ont entouré la création d'une œuvre.

\section{À la recherche d'une définition stable}

L'originalité a été traditionnellement définie comme «l'empreinte de la personnalité de l'auteur». À l'origine, cette expression a été forgée par la jurisprudence au $\mathrm{XIX}^{\mathrm{e}}$ siècle, à l'occasion des premiers contentieux concernant la photographie naissante ${ }^{13}$. Elle a ensuite été consacrée par la doctrine, notamment dans le célèbre traité du professeur Desbois ${ }^{14}$. Puis cette conception de l'originalité s'est révélée peu à peu inadaptée à des créations utilitaires ou techniques qui sortent du champ des beauxarts tels les logiciels ou les bases de données. Elle a évolué, sous l'influence du droit de l'Union européenne, pour accueillir dans le champ du droit d'auteur ces nouveaux types de créations. Aujourd'hui, une nouvelle conception de l'originalité vient concurrencer la conception historique : est appréhendée comme originale la « création intellectuelle propre à son auteur ». Cette expression provient de trois directives sectorielles qui ont harmonisé la définition de l'originalité pour les photographies, les logiciels et les bases de données. S'inspirant de ces précédents, la Cour de justice des communautés européennes (CJCE) a estimé dans sa décision «Infopaq I 》 de $2009{ }^{15}$ que l'originalité est une notion autonome du droit de l'Union, applicable à toutes les œuvres. Cela signifie qu'elle est compétente pour donner un sens commun à l'expression « création intellectuelle propre à son auteur ", qui s'impose à tous les États membres de l'Union européenne.

Par ailleurs, devant la difficulté de définir positivement l'originalité, il est utile de raisonner à rebours en identifiant trois séries de critères qui sont indifférents pour l'apprécier concrètement. D'abord, ni la quantité de travail fournie ni le savoir-faire ne doivent entrer en compte. Deuxièmement, la loi interdit de discriminer les œuvres selon le genre, la forme d'expression, le mérite ou la destination ${ }^{16}$. Enfin, l'expressivité de l'auteur dans son œuvre s'analyse à la lueur d'une opposition sous-jacente entre liberté et déterminisme: lorsqu'une création est entièrement déterminée par des contraintes extérieures qui s'imposent à l'auteur, il n'y a plus de place pour des choix individuels.

\section{La démonstration de l'originalité}

Il n'existe en droit français aucune présomption légale ou jurisprudentielle d'originalité mais dans la pratique contractuelle, l'œuvre se trouve de facto présumée originale par les parties tant que l'originalité n'est pas contestée.

En théorie, l'originalité ne se décrète pas ; elle doit être établie. Conformément au droit commun, la charge de la preuve repose sur celui qui invoque l'originalité de sa création ${ }^{17}$. Cependant, il en va autrement dans les faits. D'une part, pendant longtemps, il existait en jurisprudence une forme de présomption d'originalité des œuvres tant qu'elle n'était pas contestée par le défendeur. Les tribunaux n'avaient à examiner la question de l'originalité que pour des œuvres se situant aux confins de la protection telles que les créations utilitaires et certaines photographies. D'autre part, comme l'écrivent les professeurs Lucas dans leur Traité de la propriété littéraire et artistique :

Tout se passe souvent en pratique comme si l'œuvre bénéficiait d'une présomption

d'originalité. [...] C'est seulement dans les cas limites où la nature de l'œuvre fait 
douter de la possibilité de la protection, par exemple sur des œuvres scientifiques ou techniques, que le débat sur l'originalité revient au premier plan et que les règles de droit commun sur la charge de la preuve reçoivent application ${ }^{18}$.

17 À la faveur des fulgurantes mutations numériques, qui accroissent tant les possibilités de création que de diffusion des œuvres, et par conséquent les potentiels litiges, la position de la jurisprudence a évolué concernant la charge de la preuve de l'originalité. Les tribunaux sont devenus plus exigeants à l'égard du demandeur d'une action en contrefaçon, qui doit démontrer l'originalité de son œuvre. Afin de faire le point sur les difficultés que cela pose, notamment dans le cadre de contentieux sur un grand volume d'œuvres, où l'originalité doit être établie au cas par cas, et d'envisager d'éventuels moyens d'y remédier, le Conseil supérieur de la propriété littéraire et artistique (CSPLA) conduit actuellement une mission sur la question de la preuve de l'originalité ${ }^{19}$.

\section{La directive de 2019 sur le droit d'auteur}

Sur le plan théorique, il convient par ailleurs de tenir compte d'une nouvelle disposition du droit de l'Union européenne qui envisage précisément la question de la reproduction des œuvres d'art visuel du domaine public. L'article 14 de la directive (UE) 2019/790 du Parlement européen du 17 avril 2019 sur le droit d'auteur et les droits voisins dans le marché unique numérique et modifiant les directives 96/9/CE et 2001/29/CE envisage cette question des œuvres d'art visuel dans le domaine public: «Les États membres prévoient que, lorsque la durée de protection d'une œuvre d'art visuel est arrivée à expiration, tout matériel issu d'un acte de reproduction de cette œuvre ne peut être soumis au droit d'auteur ni aux droits voisins, à moins que le matériel issu de cet acte de reproduction ne soit original, en ce sens qu'il est la création intellectuelle propre à son auteur. »

19 Comment interpréter cette disposition qui semble tautologique, affirmant que n'est protégé par le droit d'auteur que ce qui est original ? La lecture du considérant 53 de la directive montre qu'elle s'attaque au cas des reproductions fidèles à l'œuvre de départ, et de ce fait, dépourvues d'originalité :

En matière d'arts visuels, la circulation de reproductions fidèles d'œuvres dans le domaine public contribue à l'accès à la culture et à sa promotion et à l'accès au patrimoine culturel. [...] Certaines reproductions d'œuvres d'art visuel dans le domaine public ne devraient, dès lors, pas être protégées par le droit d'auteur et les droits voisins.

L'article 14 de la directive s'avère être le fruit d'un compromis politique visant à répondre aux critiques dressées par les défenseurs du domaine public et des « communs $^{20}$ " qui dénoncent comme «copyfraud ${ }^{21}$ » certaines pratiques de réappropriation indue d'éléments du domaine public. À notre sens, elle ne fait que confirmer l'état du droit actuel, établi par la directive de 2006 précitée, et ne remet pas en cause l'éventuelle protection de certaines numérisations d'œuvres d'art. Il s'agit d'empêcher la création de nouveaux droits dans les cas de reproductions complètement automatisées tout en réservant le cas particulier de celles qui ne sont pas de simples copies du réel mais des œuvres originales à part entière. La difficulté de ce débat tient au fait que l'originalité s'apprécie œuvre par œuvre et non par catégorie générale. Le texte de la directive ne peut donc écarter définitivement l'idée qu'une reproduction photographique d'une œuvre du domaine public puisse être protégée par le droit 
d'auteur. D'une certaine manière, elle rappelle l'obligation de démontrer in concreto qu'on est bien en présence d'une « création intellectuelle propre à son auteur ».

\section{Le débat sur l'originalité en matière de photographie}

\section{Une harmonisation des critères de protection par le droit européen}

Dans la mesure où il n'existe, pour l'heure, que très peu de jurisprudence sur la numérisation tridimensionnelle, on se propose ici de raisonner par analogie à l'appui d'autres exemples de créations protégées afin d'en tirer des enseignements. À cet égard, les réflexions relatives à la numérisation en deux dimensions, en particulier via la photographie, sont éclairantes.

L'histoire des rapports de la photographie avec le droit d'auteur témoigne de la difficulté de trouver le bon critère de protection parce qu'il lui a toujours été reproché soit son caractère technique, soit sa reproduction littérale du réel. Avant une loi de $1985^{22}$, seules étaient protégées en France les photographies à « caractère artistique ou documentaire». Ce statut spécial fut critiqué en raison de la casuistique jurisprudentielle à laquelle il donna lieu, à tel point que la condition a été abandonnée, au profit d'un retour au droit commun: les photographies sont protégées comme toutes les autres œuvres.

En outre, la directive 2006/116/CE relative à la durée de protection du droit d'auteur et de certains droits voisins a harmonisé la protection des photographies en Europe. Son article 6 dispose que «Les photographies qui sont originales en ce sens qu'elles sont une création intellectuelle propre à leur auteur sont protégées conformément à l'article $1^{\mathrm{er}}$. Aucun autre critère ne s'applique pour déterminer si elles peuvent bénéficier de la protection. Les États membres peuvent prévoir la protection d'autres photographies.» Plusieurs États membres comme l'Allemagne ou l'Italie protègent ainsi non seulement les photographies mais également les "simples photographies » qui ne sont pas originales ${ }^{23}$. Cela peut couvrir par exemple les clichés générés automatiquement, c'est-à-dire sans intervention humaine, par des systèmes de surveillance vidéo ou des satellites.

24 En 2010, la Cour de justice de l'Union européenne (CJUE) a rendu une décision majeure sur le fondement de la directive de 2006 en jugeant qu'une photographie est susceptible d'être protégée par le droit d'auteur, à condition «qu'elle soit une création intellectuelle de l'auteur reflétant la personnalité de ce dernier et se manifestant par les choix libres et créatifs de celui-ci lors de la réalisation de cette photographie ${ }^{24} »$. Tel est donc, aujourd'hui, le critère d'appréciation pertinent qui s'impose en Europe : il convient d'évaluer si et dans quelle mesure l'auteur a effectué des choix libres et créatifs.

Un second apport important de cette décision tient à la distinction de différentes phases du travail de photographe qui permettent de déceler l'originalité, en particulier lors de la post-production numérique :

Au stade de la phase préparatoire, l'auteur pourra choisir la mise en scène, la pose de la personne à photographier ou l'éclairage. Lors de la prise de la photographie [...] il pourra choisir le cadrage, l'angle de prise de vue ou encore l'atmosphère créée. Enfin, lors du tirage du cliché, l'auteur pourra choisir parmi diverses techniques de développement qui existent celle qu'il souhaite adopter, ou encore procéder, le cas échéant, à l'emploi de logiciels. 
dernier stade nous semble particulièrement important en matière de numérisation tridimensionnelle : bien que l'objectif soit de créer un double fidèle au réel, l'originalité peut résider en aval dans les choix qui sont faits, à l'aide d'un logiciel, en matière d'apparence ou de texture graphique.

\section{Des solutions judiciaires contrastées sur les photographies de tableaux}

Le cas des photographies de tableaux témoigne des difficultés d'apprécier l'originalité. Traditionnellement, les institutions culturelles, publiques comme privées, affirment l'existence de droits d'auteur sur les photographies des objets de leurs collections et se font céder les droits en conséquence, de la part de leurs agents ou de prestataires extérieurs. Cette qualification juridique, si elle a été relativement peu contestée devant le juge, est néanmoins critiquée par une partie de la doctrine ${ }^{25}$ et du grand public dans la mesure où elle a pour conséquence d'entraver l'exploitation d'images d'œuvres du domaine public.

peut légitimement s'interroger sur l'originalité de photographies conçues pour restituer fidèlement une œuvre préexistante tombée dans le domaine public. Toutefois, il n'est pas possible d'exclure purement et simplement la présence d'originalité ; tout est affaire de cas d'espèce selon les circonstances de la création. Dès lors qu'un photographe s'écarte d'un objectif de servilité par rapport à l'objet reproduit et exprime sa sensibilité, les tribunaux admettent la protection du droit d'auteur. La jurisprudence oscille ainsi entre deux tendances, certaines décisions rejetant l'originalité au motif que le savoir-faire technique du photographe est mis au service d'une restitution objective de l'œuvre tandis que d'autres la reconnaissent, soulignant que le photographe a délibérément opéré certains choix de cadrage ou d'éclairage.

Pour illustrer la première tendance, mentionnons une décision de $2005^{26}$, par laquelle la cour d'appel de Paris a décidé qu'une photographie de tableau n'est pas protégée si elle a " pour seul objectif de reproduire le tableau [...] avec le plus de fidélité possible, afin de le représenter tel que le perçoit le public ». Dans le sens inverse, la cour d'appel de Paris, dans une décision de 2009, a justifié l'application du droit d'auteur à une photographie de tableau ${ }^{27}$ en ces termes: «les choix ne procèdent pas d'une simple opération technique, mais de choix délibérément opérés par le photographe [...], selon la représentation qu'il a voulu donner de l'œuvre, de sorte que [...] le photographe ne s'efface pas devant la majesté de l'œuvre, mais veut au contraire exalter la quintessence de l'œuvre selon son propre regard et sa propre sensibilité. » La cour a apprécié les choix effectués par le photographe quant «à l'angle de prise de vue, au cadrage, à l'éclairage de manière à faire ressortir des contrastes de couleurs, des jeux d'ombres et de lumière ou au contraire l'homogénéité du tableau, à accentuer des traits, à mettre en relief un plan ».

Le cœur du problème se situe au niveau de la liberté créatrice dont a bénéficié l'auteur. La jurisprudence refuse de protéger les créations dont les circonstances de création montrent une absence de choix personnels si le créateur n'est par exemple que l'exécutant des instructions d'un tiers. La cour d'appel de Rennes a ainsi jugé en $2006^{28}$ que n'étaient pas protégées les photographies d'un produit alimentaire « réalisées sous l'entière direction du fabricant qui a fourni au photographe des indications précises " sur la position du produit et sur son entourage, à partir d'une maquette entièrement élaborée par un maquettiste et dont le montage final a été réalisé par un tiers. 


\section{Le conflit entre impératif de fidélité et marge de manœuvre créatrice} par le ministère de la Culture sur l'ouverture et la réutilisation des informations publiques numériques du secteur culturel ${ }^{29}$. Réalisé dans le cadre du plan national de numérisation et de valorisation des contenus culturels, son objectif principal est de proposer une doctrine sur la manière de concilier, en théorie comme en pratique, le droit de la propriété littéraire et artistique et le droit de l'ouverture des données publiques. Le guide aborde le statut des numérisations de contenus du domaine public en opérant une distinction entre la prise de vue photographique et la conversion automatisée au format numérique par l'intermédiaire d'une machine comme un scanner à plat. Si dans le premier cas de figure, la photographie peut être protégée pour les raisons que nous avons étudiées, le second correspond à un processus purement technique et automatique, où la marge de mancuvre de l'opérateur humain est réduite au minimum. Du point de vue du droit d'auteur, la différence réside dans la place de la personne humaine au sein du processus. Un simple acte de reproduction ne constitue pas un acte créatif donnant naissance à une nouvelle œuvre. Cette solution peut être rapprochée de celle dégagée par la jurisprudence au sujet de la restauration à l'identique d'une cuvre du domaine public ${ }^{30}$.

\section{Les solutions étrangères}

Soulignons que les difficultés liées à la reproduction d'œuvres du domaine public ne sont pas propres à la France. Une décision américaine de 1999 avait fait grand bruit concernant des reproductions de peintures du domaine public, commercialisées par la société Bridgeman ${ }^{31}$. Dans cette décision, les juges américains avaient considéré que des reproductions photographiques exactes d'images du domaine public ne pouvaient être protégées par le copyright américain parce qu'elles étaient dénuées d'originalité.

À l'inverse, en Allemagne, la Cour fédérale de justice a rendu en 2018 un arrêt validant la protection de versions numérisées d'images du domaine public ${ }^{32}$. Le contentieux a opposé le musée Reiss-Engelhorn-Museen de Mannheim à la fondation Wikimedia au sujet de la diffusion de dix-sept reproductions d'œuvres d'art sur le site Wikimedia Commons. En l'espèce, le musée exposait des peintures du domaine public et avait publié des photographies de ces œuvres dans un catalogue, après s'être fait céder ses droits par le photographe auteur des clichés. De plus, le musée interdisait aux visiteurs dans son règlement intérieur de prendre des photos des peintures exposées. Un visiteur avait photographié des œuvres du musée et avait scanné des images d'un catalogue du musée numérisé avant de poster toutes les images sur l'encyclopédie en ligne. Le musée a alors intenté une action sur le double fondement de la violation de l'interdiction de photographier et de la contrefaçon de droits d'auteur. La Cour allemande a estimé que la photographie d'une peinture peut être protégée en tant que photographie, dans la mesure où le photographe doit prendre un certain nombre de décisions lors de la prise, en ce qui concerne la position, l'éclairage, la distance, l'angle ou le cadrage. Elle en a conclu que cela constituait un « effort intellectuel personnel » protégé par la loi sur le droit d'auteur. Par conséquent, en publiant les images numérisées du catalogue en ligne, le visiteur avait violé le droit de publication conféré au photographe par la loi allemande sur le droit d'auteur. 


\section{L'originalité d'une numérisation en 3D}

\section{Copie à l'identique contre nouvelle création}

implique un impératif de fidélité. La personne physique qui a la maîtrise d'un logiciel de modélisation duplique un objet qui existe déjà dans l'univers physique. En ce sens, elle ne réaliserait pas une création personnelle et subjective mais ses choix seraient dictés par la volonté de restituer le réel. Or, du point de vue du droit d'auteur, créer signifie transformer, et non retranscrire l'existant: les copies à l'identique d'œuvres préexistantes ne sont pas protégées par le droit d'auteur. C'est pourquoi la jurisprudence française a refusé par exemple de protéger la transcription de manuscrits médiévaux ${ }^{33}$. Toutefois, il faut avoir conscience que cette ligne de partage entre créations protégées et domaine public n'est pas universelle. Au sein de l'Union européenne par exemple, l'article 5 de la directive 2006/116/CE précitée autorise les États membres à protéger «les éditions critiques et scientifiques d'œuvres qui sont tombées dans le domaine public ».

À l'inverse, on peut considérer par un raisonnement a fortiori que la marge de manœuvre créatrice est plus grande pour la numérisation d'un objet en trois dimensions. Là où un tableau laisse a priori peu de latitude pour le photographier de face, il existe de multiples manières de reproduire une statue ou une fontaine. En outre, l'ajout d'une couche d'interactivité des images peut changer la donne dès lors qu'elles ne se bornent pas à reproduire servilement l'œuvre de départ mais l'animent.

Un rapport du CSPLA de 2016, consacré à l'impression 3D ${ }^{34}$, a étudié les questions juridiques liées à l'impression d'objets physiques à partir de modèles numériques en 3D. Le rapport s'est concentré sur les œuvres protégées par le droit d'auteur et non sur la numérisation d'œuvres tombées dans le domaine public mais on peut en tirer ici plusieurs riches enseignements. Il estime, d'une part, qu'un fichier 3D créé par simple numérisation 3D d'un objet existant ne devrait pas être protégé, en l'absence de trace de l'empreinte de la personnalité de la personne ayant créé le fichier, et d'autre part, que cette analyse pourrait être infirmée dans les cas où l'acte de numérisation impliquerait des choix faits par l'utilisateur du scanner, s'agissant par exemple de la résolution de l'image ou de la luminosité. Le rapport envisage également certaines hypothèses intermédiaires,

telle celle où une œuvre serait d'abord numérisée en 3D avant que le fichier soit modifié par l'utilisation d'un logiciel de CAO (personnalisation de l'œuvre). Dans ce cas, a priori les éléments qui ne constituent pas une simple reprise de l'œuvre initiale pourraient être protégés au titre du droit d'auteur, dès lors qu'ils révèlent une création originale. Il peut donc y avoir coexistence de droits sous réserve naturellement du respect des droits, notamment moraux, de l'auteur de l'œuvre initiale. 


\section{La notion d'œuvre complexe} régime de l'œuvre collective qui est avantageux pour le promoteur du jeu, qui se voit automatiquement investi des droits des auteurs individuels. D'autres ont cherché à rapprocher le jeu vidéo d'œuvres qui lui ressemblent. Une première partie a eu recours au droit spécial du logiciel, avantageux pour un employeur dans la mesure où il bénéficie d'une dévolution automatique de ses auteurs salariés, tandis qu'une seconde est allée vers l'œuvre audiovisuelle. L'arrêt «Cryo » rendu par la première Chambre civile de la Cour de cassation le 25 juin 2009 a mis un terme à ces hésitations de la jurisprudence ${ }^{35}$. La Cour a jugé qu' « un jeu vidéo est une œuvre complexe qui ne saurait être réduite à sa seule dimension logicielle, quelle que soit l'importance de celle-ci, de sorte que chacune de ses composantes est soumise au régime qui lui est applicable en fonction de sa nature ». Le caractère protéiforme de l'œuvre complexe conduit à la mise en place d'une qualification distributive : chaque élément se voit appliquer son régime particulier.

À la lueur de ces considérations, il nous semble que certaines numérisations patrimoniales en 3D, lorsqu'elles s'insèrent dans un ensemble mêlant logiciel, photos et effets audiovisuels, peuvent être qualifiées d'œuvres complexes. Pour examiner au cas par cas si on a affaire à une création originale, deux critères sont essentiels.

41 Le premier critère, le plus déterminant, est celui de la finalité poursuivie. Une numérisation patrimoniale faite à des fins exclusives de recherche et strictement identique à l'objet de départ est bien éloignée de la condition d'originalité. En revanche, dès que cette numérisation devient partie prenante d'une création plus englobante, par exemple une animation de réalité virtuelle dans laquelle un utilisateur peut interagir ou un jeu vidéo, nous voyons bien qu'on entre là dans une œuvre dérivée à part entière. Dès lors qu'on va au-delà d'un impératif de fidélité mais que s'ajoute une " patte artistique », on peut considérer qu'il y a originalité.

42

second critère, qui découle du premier, est le résultat obtenu. Il faut souligner qu'un environnement ou un style graphique en particulier peuvent être protégés, si l'on songe par exemple aux images de la cathédrale Notre-Dame de Paris qui apparaissent dans le jeu vidéo d'Ubisoft, Assassin's Creed Unity. Des choix peuvent être effectués par l'auteur des images, par l'intermédiaire de logiciels de création. La jurisprudence française a ainsi reconnu que les images de synthèse sont protégées par le droit d'auteur dès lors qu'elles sont originales ${ }^{36}$. De plus, au plan européen, dans une affaire qui concernait l'interface utilisateur graphique d'un logiciel, la CJUE a jugé qu'une telle interface peut bénéficier de la protection du droit d'auteur si elle constitue une « création intellectuelle propre à son auteur ${ }^{37}$ ». 


\section{Les incidences pratiques : comment contrôler la numérisation patrimoniale?}

Au-delà du débat théorique complexe sur l'originalité, le droit d'auteur reste un outil contractuel solide pour encadrer par avance les usages d'un objet numérique. En pratique, faire de la numérisation 3D d'un objet patrimonial une création non protégée n'est pas satisfaisant pour l'institution culturelle qui y a consacré des investissements parce que si le droit de la propriété intellectuelle ne s'applique pas, elle devient librement réutilisable par tous. Au-delà de l'enjeu de valorisation économique, la perte de contrôle sur l'usage de l'objet soulève également des craintes concernant la continuité du contrôle scientifique et technique de l'État en matière de patrimoine.

Pour garder la main sur les numérisations 3D, la cession de droits d'auteur dans le cadre d'un marché public de numérisation passé avec la société prestataire s'avère donc en pratique très utile. La solution la plus sécurisante pour l'État consiste donc à présumer qu'on est en présence d'une œuvre originale et à se faire céder les droits nécessaires par contrat. Mais à terme, on ne pourra faire l'économie d'un travail de qualification plus poussé de ce type de création au regard du droit d'auteur.

En outre, il convient de tenir compte des pratiques contractuelles des entreprises privées proposant des services de numérisation 3D, qui considèrent qu'elles détiennent des droits d'auteur sur les fichiers de numérisation qu'elles ont réalisés. Le droit d'auteur permet ici de sécuriser les usages de la vie économique. Toutefois, dans certains cas, le prestataire peut avoir reçu des demandes précises, formulées dans un cahier des charges contraignant, de sorte qu'il ne disposait pas de liberté créatrice et ne pourra prétendre à la protection du droit d'auteur. On en revient alors à la nécessité, relevée précédemment, de démontrer que l'on est bien en présence d'une création originale.

\section{La numérisation en 3D au prisme du droit des informations publiques}

Le droit d'auteur doit aujourd'hui composer avec de nouvelles régulations, qui ne raisonnent pas en termes d'œuvres ou d'objets patrimoniaux mais en termes de données. Comme l'a souligné un rapport du CSPLA de 2018 dédié à cette question ${ }^{38}$, « Le caractère expansif tant des définitions des objets protégés par la propriété littéraire et artistique que des termes de "données » et de «contenus numériques » engendre un croisement de ces notions, source de perturbation des règles applicables. »

Dans le langage courant, on parle de l'ouverture des données publiques ou d'«open data» mais la loi utilise en réalité la notion d'informations publiques. Le flou terminologique des textes juridiques autour des notions de "données", d'« informations » ou de «documents » est d'ailleurs un indice de la difficulté pour le droit de s'adapter à la vitesse des évolutions numériques. Les règles encadrant l'usage de ces informations publiques sont rassemblées dans le Code des relations entre le public et l'administration (CRPA). À l'origine, une loi de $1978^{39}$ dite «Cada » (d'après le nom de la Commission d'accès aux documents administratifs), avait créé un droit d'accès aux documents administratifs pour tous les citoyens. Sous l'influence du droit de l'Union européenne, ce droit a ensuite été complété par un droit de réutilisation des informations contenues dans ces documents. D'une logique de l'accès, on est donc passé à une logique de la réutilisation. 

communiqués ou publiés sous réserve des droits de propriété littéraire et artistique ». La Cada considère de manière constante que cette disposition n'a ni pour objet ni pour effet d'empêcher ou de restreindre cette communication ${ }^{43}$. Autrement dit, une personne publique ne peut se fonder sur elle pour refuser la communication d'un fichier de numérisation 3D. En revanche, il convient de bien distinguer communication et réutilisation : l'administration peut dans certains cas limiter l'usage ultérieur que le demandeur, après communication, voudrait faire des documents. La personne qui se voit communiquer par l'intermédiaire d'une demande d'accès un fichier sur lequel des tiers détiennent des droits de propriété intellectuelle n'a pas le droit de le réutiliser, en dehors des cas de figure qui relèvent d'exceptions au droit d'auteur. Tel est le cas en particulier de l'exception pour copie privée, qui autorise l'usage privé d'une œuvre de l'esprit mais interdit l'utilisation collective qui pourrait en être faite ${ }^{44}$.

51 Il convient toutefois de rester vigilant quant à l'exercice du droit de divulgation, qui est une composante des droits moraux encadrée par l'article L. 121-2 du CPI : il s'agit du droit de chaque auteur de choisir quand et comment rendre son œuvre publique pour la première fois. Le Conseil d'État a jugé en 2017 que l'article L. 311-4 du CRPA implique, avant de procéder à la communication de supports d'enseignement n'ayant pas déjà fait l'objet d'une divulgation, de recueillir l'accord de leur auteur ${ }^{45}$.

\section{La réutilisation des informations publiques}

\section{Le principe et ses limites} documents administratifs communiqués ou publiés par les administrations : l'État, les collectivités territoriales, les établissements publics mais aussi les personnes de droit privé chargées d'une mission de service public.

53 La loi pose le principe que les informations publiques produites ou reçues par une administration dans le cadre de sa mission de service public peuvent être réutilisées 
par toute personne à d'autres fins, y compris à des fins commerciales. Notons ici une différence sensible de ce droit par rapport à la propriété intellectuelle: on raisonne cette fois en termes d'usage et non de propriété. L'administration ne peut contrôler en amont les usages ultérieurs de ses données qui sont faits par les citoyens ou les entreprises mais ces derniers ont une obligation, proche du droit moral au respect de l'intégrité de l'œuvre, de ne pas dénaturer ces données et de mentionner leur source ${ }^{46}$.

Cependant, les informations contenues dans un document administratif ne sont librement réutilisables que si ce document remplit deux conditions. D'abord, il doit être librement communicable à toute personne, en particulier au regard des délais de communicabilité des archives publiques prévues par le Code du patrimoine. Ensuite, et cette exception est majeure sur le sujet qui nous occupe, l'article L. 321-2 du CRPA prévoit que "ne sont pas considérées comme des informations publiques, pour l'application du présent titre, les informations contenues dans des documents : [...] c) Ou sur lesquels des tiers détiennent des droits de propriété intellectuelle».

L'interprétation de cette disposition s'avère redoutable. Concernant les droits visés, elle est classiquement comprise comme ne couvrant que les droits patrimoniaux d'un auteur, et non ses droits moraux, dans la mesure où leur perpétuité viendrait vider de sa substance le principe même de la réutilisation des informations publiques : le droit moral d'un tiers persisterait en permanence. L'esprit de la loi conduit à penser que seuls les droits exclusifs d'exploitation sont visés, c'est-à-dire le droit d'autoriser ou interdire une exploitation, et non des droits rattachés à la personne de l'auteur. Concernant les tiers, en revanche, l'analyse est plus difficile.

\section{Qui sont les tiers à l'administration visés par la loi ?}

Deux cas de figure doivent ici être distingués. Une numérisation tridimensionnelle peut avoir été réalisée soit par les propres agents de l'administration, soit par un prestataire extérieur, par exemple dans le cadre d'un marché public.

En premier lieu, les articles L.131-3-1 et suivants du CPI aménagent un régime particulier pour les droits d'exploitation de certains agents publics, qui ont créé des œuvres dans l'exercice de leurs fonctions ou d'après les instructions reçues par leur employeur. Dans la mesure strictement nécessaire à l'accomplissement d'une mission de service public, le droit d'exploitation d'une œuvre créée par un agent est, dès sa création, cédé de plein droit à l'État ${ }^{47}$. Néanmoins, pour une exploitation commerciale de l'œuvre, l'auteur recouvre la titularité de ses droits, et l'employeur public ne dispose envers l'agent auteur que d'un droit de préférence: avant tout projet d'exploitation commerciale, l'agent auteur devra se tourner en priorité vers son employeur public. Ce régime ne s'applique pas à tous les agents publics, l'article L. 111-1 du CPI excluant expressément du champ des articles L. 131-3-1 et suivants les "agents auteurs d'œuvres dont la divulgation n'est soumise, en vertu de leur statut ou des règles qui régissent leurs fonctions, à aucun contrôle préalable de l'autorité hiérarchique ». Il s'agit en particulier des chercheurs, enseignants chercheurs dont le statut garantit l'indépendance et la liberté de création dans leur activité de production des connaissances. Par conséquent, identifier qui est éventuellement titulaire des droits d'auteur sur une numérisation tridimensionnelle réalisée par des agents publics implique d'avoir clarifié en amont le statut de ces agents. 

d'utilisation par la Poste d'une photographie réalisée par un agent du service régional de l'Inventaire du patrimoine culturel, que « hors le cas où les droits d'exploitation auraient été cédés contractuellement à l'administration par l'agent dans les conditions de droit commun du Code de la propriété intellectuelle, les informations contenues dans des documents sur lesquels des agents publics détiennent des droits de propriété intellectuelle ne peuvent, en l'état actuel des textes, être regardées comme des informations publiques au sens de l'article 10 de la loi du 17 juillet $1978^{48}$.». Ce faisant, elle a considéré que les auteurs agents publics constituent des tiers à l'administration au sens de l'article L. 321-2 du CRPA.

En second lieu, une institution culturelle a pu commander une numérisation tridimensionnelle à un prestataire privé. Dans ce cadre, les documents du marché public prévoient en général une cession de droits d'auteur sur les résultats du marché, qui doit respecter les mentions obligatoires et le formalisme prévus par l'article L. 131-3 du CPI pour toutes les transmissions de droits d'auteur. À cet égard, dans tout marché public qui comporte des prestations intellectuelles, il est recommandé de s'appuyer sur le cadre de référence qu'est le Cahier des clauses administratives générales applicables aux marchés publics de prestations intellectuelles ${ }^{49}$ (CCAG-PI) de 2009. L'article 25 du CCAG-PI, qui offre à une personne publique deux possibilités pour acquérir des droits d'auteur sur les résultats livrés par le titulaire d'un marché public, prévoit une "option $\mathrm{B}$ » consistant en une cession à titre exclusif des droits sur les résultats du marché. En d'autres termes, le titulaire cède les droits d'exploitation des résultats au maitre d'ouvrage, qui peut alors les exploiter et les céder à des tiers, pour les seuls modes d'exploitation prévus au marché.

Il faut donc avoir conscience que lorsqu'une institution culturelle publique est la seule titulaire de tous les droits de propriété intellectuelle sur une numérisation tridimensionnelle, il n'existe pas de droits détenus par des tiers au sens de l'article L. 321-2 du CRPA. Cela signifie qu'elle ne peut se prévaloir de ses propres droits pour faire obstacle à l'application du droit de la réutilisation des informations publiques. Seule la présence de droits appartenant à des tiers fait sortir du champ des informations publiques les fichiers concernés pour les faire relever du droit d'auteur, qui subordonne l'utilisation d'un fichier de numérisation à une autorisation préalable. C'est ici qu'intervient une difficulté de conciliation de l'exception à l'open data pour les documents sur lesquels des tiers détiennent des droits de propriété intellectuelle avec le formalisme strict des cessions de droit d'auteur. Paradoxalement, pour pouvoir empêcher la réutilisation d'une numérisation protégée, une institution culturelle a intérêt à ne se faire céder qu'une partie des droits de la société prestataire. Il convient d'être vigilant et précis dans la rédaction des cessions de droits d'auteur, en particulier s'agissant de l'étendue et de la destination des droits cédés en accord avec l'article L. 131-3 du CPI.

\section{Un premier cas concernant la numérisation 3D soumis à la Cada}

La Cada s'est prononcée le 7 février 2019, pour la première fois, au sujet d'une question relative à la numérisation en trois dimensions du patrimoine ${ }^{50}$. Elle avait été saisie par le musée Rodin d'une demande de conseil sur plusieurs questions: le caractère communicable des numérisations tridimensionnelles d'œuvres d'art, au regard de la 
qualité d'ayant droit de l'artiste du musée; la possibilité de réutiliser à des fins commerciales ces numérisations, au regard du droit de la propriété intellectuelle et du risque de contrefaçon; enfin, la possibilité de réutiliser des numérisations tridimensionnelles, considérant le mode de financement du musée ${ }^{51}$.

62 Au terme de son examen, la commission a décidé que les numérisations d'œuvres dont le musée Rodin assure la conservation, à des fins à la fois d'étude et d'exploitation commerciale, constituent des documents administratifs au sens du CRPA, dès lors que le musée les a élaborées et les détient dans le cadre de la mission de service public qui lui est confiée. Elles sont donc, en principe, communicables à toute personne qui en fait la demande, sur le fondement de l'article L. 311-1 du CRPA, sous réserve qu'elles ne présentent plus un caractère préparatoire à une décision que le musée n'aurait pas encore prise.

63 En outre, la Cada a estimé que ni les considérations tirées du mode de financement de l'établissement public qui sont inopérantes en matière de communication des documents administratifs, ni la qualité d'ayant droit de Rodin, chargé de faire respecter le droit moral du sculpteur, ne sont susceptibles de fonder un refus de communication, à charge pour le musée de veiller au respect de l'œuvre lors de la réutilisation des documents sollicités.

64 Cette décision inédite soulève des enjeux importants pour les institutions culturelles, qui concernent à la fois l'articulation entre leurs missions de service public, la poursuite de missions commerciales et le développement de ressources propres. Il conviendra d'en suivre attentivement les conséquences.

\section{Un principe général de gratuité de la réutilisation, mais une dérogation pour le secteur culturel}

La loi prévoit un principe de gratuité de la réutilisation des données publiques. La tarification est l'exception et n'est autorisée que deux cas, dont l'un vise directement les institutions culturelles. La réutilisation peut donner lieu au versement d'une redevance lorsqu'elle porte sur "des informations issues des opérations de numérisation des fonds et des collections des bibliothèques, y compris des bibliothèques universitaires, des musées et des archives et, le cas échéant, sur des informations qui y sont associées lorsque ces dernières sont commercialisées conjointement ${ }^{52}$ ».

Dans le cas de la réutilisation d'un fichier numérique de numérisation 3D, ce texte constitue un fondement sur lequel peut s'appuyer une institution culturelle pour demander au réutilisateur une redevance. La loi prévoit que le produit total du montant de cette dernière, évalué sur une période comptable appropriée, ne dépasse pas le montant total des coûts de collecte, de production, de mise à disposition ou de diffusion, de conservation de leurs informations et d'acquisition des droits de propriété intellectuelle ${ }^{53}$.

Il n'y a pas d'obligation pour les administrations de fixer une licence pour la réutilisation d'informations publiques à titre gratuit. Pour éviter une prolifération des licences, un décret de $2017^{54}$ offre le choix entre deux licences : la Licence ouverte, conçue par Etalab, le service interministériel qui coordonne la politique d'ouverture des données publiques, et la licence OdbL (Open Database License) pour les bases de 
données ${ }^{55}$. En revanche, quand des données publiques sont mises à disposition contre le paiement d'une redevance, les administrations sont dans l'obligation de fixer une licence et de mettre une ou des licences types, par voie électronique, à la disposition des personnes intéressées par la réutilisation de ces informations. Dans son avis du 7 février 2019 précité, la Cada a indiqué qu'un musée peut établir une tarification pour la réutilisation de ses numérisations tridimensionnelles, qu'elle doit publier sur son site internet, conformément aux dispositions du CRPA. Conclusion

La numérisation tridimensionnelle d'objets du patrimoine soulève de nombreuses questions, au croisement de la propriété intellectuelle et des données publiques. À ce stade, s'il n'existe pas de réponses définitives, des pistes de solution émergent dans les pratiques, notamment contractuelles. D'une manière générale, il convient d'être au clair sur l'objet dont on parle: sous les termes "numérisation en 3D " se nichent plusieurs procédés techniques. Qu'il s'agisse des milliers de photographies prises par photogrammétrie, d'un nuage de points généré par lasergrammétrie, d'un modèle réalisé à partir d'un logiciel de conception ou encore d'une animation de visite virtuelle, les créations qui en résultent ne relèvent pas forcément des mêmes régimes juridiques. La solution doit être affinée selon les différents types de numérisation qui sont en jeu.

Le droit d'auteur trace une ligne de partage entre la créativité humaine, fût-elle minimale, et l'automatisation technique. Parfois poreuse, cette frontière évolue en fonction de l'interprétation conduite par les tribunaux et au gré des diverses techniques de numérisation employées. Quant au droit des informations publiques, il pose la question de la place de la numérisation au sein des institutions culturelles publiques à un double titre. Il les amène à articuler de la meilleure manière possible, d'une part leurs missions de service de public et leurs activités commerciales, et d'autre part l'application éventuelle du droit d'auteur en amont, à travers les cessions des droits des auteurs, et la réutilisation permise en aval, qui peut favoriser à son tour la création d'œuvres dérivées.

\section{NOTES}

1. Ces différents enjeux, qui font l'objet de travaux réguliers, ont été abordés en particulier dans le rapport "Musées du Xxi ${ }^{e}$ siècle » remis en 2017 par Jacqueline Eidelman à la ministre de la Culture et de la Communication Audrey Azoulay: http://www.culture.gouv.fr/Espacedocumentation/Rapports/Rapport-de-la-mission-Musees-du-XXIe-siecle. Puis ils ont fait l'objet d'une journée professionnelle, organisée le 5 octobre 2018 par la direction générale des Patrimoines du ministère, sur «La stratégie numérique dans les musées »: http:// www.culture.gouv.fr/Thematiques/Musees/Nos-actions/Colloques-Journees-d-etudes/Journeeprofessionnelle-La-strategie-numerique-dans-les-musees-programme.

2. Décret no 2017-1077 du 24 mai 2017 relatif aux attributions du ministre de la Culture.

3. Le domaine public ne fait pas l'objet d'une définition positive en droit français. Il désigne l'ensemble des œuvres qui ne sont plus protégées au titre du droit d'auteur, une fois le délai de 
protection écoulé, à savoir soixante-dix ans après la mort de l'auteur. On peut également y inclure tous les éléments qui ne sont pas susceptibles d'être appropriés, tels les faits, les données brutes, les méthodes, les idées, et les créations non originales.

4. Mentionnons en particulier plusieurs rapports du Conseil supérieur de la propriété littéraire et artistique (CSPLA) : sur le domaine commun informationnel en 2016, l'impression 3D en 2016 et les licences libres dans le secteur culturel en 2017. Tous ces rapports sont publiés sur le site internet du CSPLA : http://www.culture.gouv.fr/Thematiques/Propriete-litteraire-et-artistique/ Conseil-superieur-de-la-propriete-litteraire-et-artistique.

5. Une visite virtuelle de la grotte Chauvet-Pont d'Arc est ainsi possible à cette adresse : http:// archeologie.culture.fr/chauvet/fr.

6. Les différentes collections 3D sont accessibles en ligne à cette adresse: https:// www.photo.rmn.fr/C.aspx?VP3=CMS3\&VF=RMNR5N_92_Vform\&FRM=Frame:RMNR5N_95.

7. https://sketchfab.com/francecollections

8. http://archeologie.culture.fr/palmyre/fr

9. Depuis la loi $n^{\circ} 2016-925$ du 7 juillet 2016 relative à la liberté de la création, à l'architecture et au patrimoine (LCAP), cette définition a été complétée par un second alinéa pour accueillir le " patrimoine culturel immatériel », encadré par la convention de l’Unesco de 2003.

10. Article L. 113-2, deuxième alinéa, du CPI.

11. Article L. 112-3 du CPI.

12. Article L. 112-2 du CPI.

13. En particulier dans une affaire qui concernait la reproduction d'un portrait du comte de Cavour : cour d'appel de Paris, 10 avril 1862, D.1863.I.53. Sur la reconnaissance de la photographie comme art protégé par le droit d'auteur, voir: GIRARDIN Daniel \& PIRKER Christian (dir.), Controverses. Une histoire juridique et éthique de la photographie, Arles / Lausanne, Actes Sud / musée de l'Élysée, 2008.

14. DESBOIS Henri, Le Droit d'auteur en France, Paris, Dalloz, 1978 [1966].

15. CJCE, 16 juillet 2009, affaire C-5/08, Infopaq International A/S / Danske Dagblades Forening.

16. Article L. 112-1 du CPI.

17. En application de l'article 1353 du Code civil, qui dispose que «Celui qui réclame l'exécution d'une obligation doit la prouver ».

18. LUCAS André \& LUCAS Henri-Jacques, Traité de la propriété littéraire et artistique, Paris, Litec, 2006 [1994], nº 85.

19. http://www.culture.gouv.fr/Thematiques/Propriete-litteraire-et-artistique/Conseilsuperieur-de-la-propriete-litteraire-et-artistique/Travaux/Missions/Mission-du-CSPLA-sur-lapreuve-de-l-originalite

20. Désignant à l'origine des ressources naturelles telles que des forêts ou des pâturages dont l'usage est partagé, la notion a été étendue dans la seconde moitié du $\mathrm{xx}^{\mathrm{e}}$ siècle à la sphère immatérielle pour appréhender les informations et connaissances qui sont diffusées et partagées sous forme numérique, en particulier sous l'impulsion des travaux d'Elinor Ostrom sur la gouvernance économique des biens communs, couronnés en 2009 par le «prix Nobel» d'économie.

21. Cette notion a été étudiée dans le rapport au CSPLA de FARCHY Joëlle \& DE LA TAILLE Marie, «Les licences libres dans le secteur culturel», 2017. Disponible en ligne, https:// www.culture.gouv.fr/Sites-thematiques/Propriete-litteraire-et-artistique/Conseil-superieur-dela-propriete-litteraire-et-artistique/Travaux/Missions/Mission-du-CSPLA-sur-l-economie-deslicences-libres-dans-le-secteur-culturel [lien valide en avril 2020].

22. Loi $\mathrm{n}^{\circ} 85-660$ du 3 juillet 1985 relative aux droits d'auteur et aux droits des artistesinterprètes, des producteurs de phonogrammes et de vidéogrammes et des entreprises de communication audiovisuelle. 
23. Ainsi, en vertu de l'article 72 de la loi allemande sur le droit d'auteur, même dans des cas où elle témoigne d'une créativité minimale, une "simple photographie " ( Lichtbildwerk») peut bénéficier de la protection prévue pour les «œuvres photographiques» si elle témoigne d'un niveau minimal d'« effort intellectuel personnel ». Toutefois, une durée de protection plus courte est prévue pour ces « simples photographies».

24. Cour de justice de l'Union européenne, $1^{\mathrm{er}}$ décembre 2010, affaire C-145/10, Eva-Maria Painer c / Standard VerlagsGmbH e.a.

25. Voir LATREILLE Antoine, "L'appropriation des photographies d'œuvres d'art ", Dalloz, 2002, chron. p. 299.

26. CA Paris, $4^{\mathrm{e}}$ ch. $\mathrm{B}, 24$ juin $2005, \mathrm{n}^{\circ} 03 / 11140$.

27. CA Paris, $4^{\mathrm{e}} \mathrm{ch}$. A, 4 mars $2009, \mathrm{n}^{\circ} 07 / 12226$.

28. CA Rennes, $2^{\mathrm{e}}$ ch., 23 mai $2006, \mathrm{n}^{\circ} 04 / 07667$.

29. Ce guide est disponible à l'adresse suivante: http://www.culture.gouv.fr/Thematiques/ Innovation-numerique/Numerisation2.

30. Voir notamment : Cour d'appel de Paris, 5 octobre 1994, D. 1996. En l'espèce, la cour a estimé que la restauration d'un film ancien ne fait pas naître de droit d'auteur sur la version restaurée, dès lors qu'il s'agit d'une opération technique et non d'un acte de création.

31. United States District Court for the Southern District of New York, Bridgeman Art Library v. Corel Corp., 36 F. Supp. 2d 191 (SDNY 1999), February 18, 1999. "In this case, plaintiff by its own admission has labored to create "slavish copies" of public domain works of art. While it may be assumed that this required both skill and effort, there was no spark of originality-indeed, the point of the exercise was to reproduce the underlying works with absolute fidelity. Copyright is not available in these circumstances. "

32. Bundesgerichtshof, Urteil vom 20.12.2018 - I ZR 104/17.

33. TGI Paris $3^{\mathrm{e}}$ chambre, $4^{\mathrm{e}}$ section, 27 mars 2014.

34. JAPIOT Olivier \& LIGNEREUX Bastien, «L'impression 3D et le droit d'auteur : des menaces à prévenir, des opportunités à saisir ", rapport de la Commission de réflexion sur l'impression 3D au Conseil supérieur de la propriété littéraire et artistique, 2016. Disponible en ligne, https:// www.google.com/search?client=firefox-b-d\&q=JAPIOT+Olivier\%2C+LIGNEREUX+Bastien\%2C+ $\% \mathrm{C} 2 \% \mathrm{AB}+\mathrm{L} \% \mathrm{E} 2 \% 80 \% 99 \mathrm{impression}+3 \mathrm{D}+\mathrm{et}+\mathrm{le}+\mathrm{droit}+\mathrm{d} \% \mathrm{E} 2 \% 80 \% 99$ auteur+\%3A+des+menaces+ $\%$ C3\%A0+pr\%C3\%A9venir\%2C+des+opportunit\%C3\%A9s+\%C3\%A0+saisir+\%C2\%BB\%2C\# [lien valide en avril 2020].

35. Cour de cassation, Chambre civile 1, 25 juin 2009, 07-20.387.

36. TGI Paris, 22 mars 1989.

37. CJUE, 22 décembre 2010, affaire C-393/09, Bezpečnostní softwarová asociace - Svaz softwarové ochrany c/ Ministerstvo kultury.

38. CSPLA, BENABOU Valérie-Laure, ZOLYNSKI Célia \& CYTERMANN Laurent, « Droit de la propriété littéraire et artistique, données et contenus numériques ", septembre 2018, p. 5. Disponible en ligne, http://www.culture.gouv.fr/Thematiques/Propriete-litteraire-et-artistique/ Conseil-superieur-de-la-propriete-litteraire-et-artistique/Travaux/Missions/Mission-du-CSPLAsur-les-consequences-pour-la-propriete-litteraire-et-artistique-de-l-avenement-des-notions-dedonnees-et-de-contenus-numeriques [lien valide en mai 2020].

39. Loi $\mathrm{n}^{\circ} 78-753 \mathrm{du} 17$ juillet 1978 portant diverses mesures d'amélioration des relations entre l'administration et le public et diverses dispositions d'ordre administratif, social et fiscal.

40. Loi $\mathrm{n}^{\circ}$ 2015-1779 du 28 décembre 2015 relative à la gratuité et aux modalités de la réutilisation des informations du secteur public.

41. Loi $n^{\circ}$ 2016-1321 du 7 octobre 2016 pour une République numérique.

42. La loi en donne une liste non exhaustive très large: dossiers, rapports, études, comptes rendus, procès-verbaux, statistiques, instructions, circulaires, notes et réponses ministérielles, correspondances, avis, prévisions, codes sources et décisions. 
43. Cada, conseil $n^{\circ} 20091473$, séance du 30 avril 2009.

44. Article L. 122-5 du CPI.

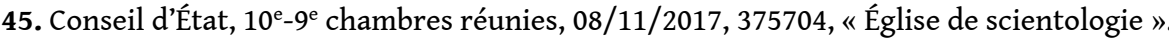

46. Toute réutilisation est soumise aux conditions suivantes: ne pas altérer les informations en question ni en dénaturer le sens, et mentionner leurs sources et la date de leur dernière mise à jour.

47. Cette disposition vise non seulement les agents employés par l'État mais aussi par les collectivités territoriales, les établissements publics à caractère administratif, les autorités administratives indépendantes dotées de la personnalité morale et la Banque de France. En revanche, les auteurs d'œuvres d'établissements publics à caractère industriel et commercial sont soumis au droit commun de la propriété littéraire et artistique.

48. Cada, avis $n^{\circ} 20092706$, séance du 5 novembre 2009.

49.

https://www.legifrance.gouv.fr/affichTexte.do?

cidTexte=JORFTEXT000021158419\&dateTexte $=\&$ categorieLien $=i d$

50. Cada, avis $\mathrm{n}^{\circ} 20190026$, séance du 27 février 2019.

51. Le mode de financement du musée Rodin est encadré par l'article 12 du décret $n^{\circ} 93-163$ du 2 février 1993.

52. Article L. 324-2 du CRPA.

53. Article L. 324-2 du CRPA.

54. Décret $n^{\circ} 2017-638$ du 27 avril 2017 relatif aux licences de réutilisation à titre gratuit des informations publiques et aux modalités de leur homologation.

55. Lorsqu'aucune licence prévue dans le décret ne répond aux besoins particuliers d'une administration et qu'elle souhaiterait recourir à une licence spécifique, elle devra en demander préalablement l'homologation par l'État après avoir adressé une demande motivée à la direction interministérielle du Numérique et du Système d'information et de communication de l'État (DINSIC).

\section{RÉSUMÉS}

La numérisation du patrimoine prend une place grandissante pour les institutions culturelles comme les musées, les services d'archives ou les bibliothèques, afin de faire évoluer leurs missions traditionnelles de conservation et de recherche mais aussi de développer de nouveaux moyens de valoriser le patrimoine. Lorsqu'un musée réalise un modèle numérique 3D d'un objet de ses collections, se posent de multiples questions juridiques sur le statut de ces nouvelles images : quels sont les droits de propriété ou d'usage sur la numérisation 3D, qui les détient et pour quoi faire?

$\mathrm{Au}$-delà du droit du patrimoine, d'autres facettes du droit ont vocation à s'entrecroiser pour saisir la numérisation en trois dimensions du patrimoine. L'idée est de se concentrer ici sur les deux principales: le droit d'auteur, concernant la protection des différentes créations numériques; le droit des informations publiques, sur l'ouverture et la réutilisation des données par les administrations («open data »).

Pour le droit d'auteur, l'enjeu est de taille: face à l'explosion du nombre de créations numériques, comment distinguer celles qu'il protège comme œuvres de l'esprit de celles qui ne peuvent faire l'objet d'une protection? Quel est le statut des modèles 3D qui visent à dupliquer 
fidèlement des objets tombés dans le domaine public? Il importe d'examiner au cas par cas comment sont conçus et réalisés les modèles pour voir s'ils présentent un caractère d'originalité, et partant, peuvent prétendre au droit d'auteur.

Par ailleurs, le droit d'auteur doit être combiné aujourd'hui avec de nouvelles régulations, qui ne portent pas sur des œuvres mais sur les données numériques. Au sens du Code des relations entre le public et l'administration, un modèle 3D détenu par une institution culturelle est qualifié de «document administratif » et les données qu'il contient sont des « informations publiques », dès lors qu'elles ne font pas l'objet de droits de propriété intellectuelle détenus par des tiers. L'application de ces notions emporte des conséquences importantes pour le secteur culturel, en termes de mise à disposition des fichiers numériques, de contrôle des usages, de licence d'utilisation et de tarification.

The digitisation of heritage plays an increasingly important role in cultural institutions such as museums, archives services or libraries, as it lets their traditional missions of conservation and research evolve, and it develops new ways of interpreting heritage. When a museum creates a digital 3D model of an object from its collections, this raises many legal issues regarding the status of these new images: what are the property rights and rights of use on 3D digitisation, who has them, and for what purpose?

Beyond heritage rights, other types of rights tend to be involved during the seizing of 3D digitisation of heritage. Our goal is to focus on the two main ones: copyright, which deals with the protection of the different digital creation; the right to public information, which deals with the release and the reutilisation of data by administration (open data).

Regarding copyright, an extremely important questions must be answered: with the explosion of the number of digital creations, how can we distinguish between those that are protected as intellectual works, and those that cannot have such protection? What is the status of 3D models that are meant to faithfully duplicate objects that have fallen into the public domain? We must examine each case individually, see how models are conceived and created, to determine whether or not they are original works and therefore are eligible for copyrights.

However, copyright must now ne combined with new regulations, which do not deal with works, but with digital data. According to the code des relations entre le public et l'administration, a 3D model owned by a cultural institution is an "administrative document " and the data it contains are "public information", as long as they are not subjected to intellectual property rights by others. The application of those notions involves important consequences to the cultural sector, regarding how digital files are made available to the public, control of usages, and licence of use and pricing.

\section{INDEX}

Keywords : Copyright, digitisation, public domain, public data, open data, heritage, photography, reproduction, distribution, reutilisation

Mots-clés : droit d'auteur, numérisation, domaine public, données publiques, open data, patrimoine, photographie, reproduction, diffusion, réutilisation

\section{AUTEUR}

\section{SAMUEL BONNAUD-LE ROUX}

Adjoint à la cheffe du bureau de la Législation, Secrétariat général du ministère de la Culture samuel.bonnaud-le-roux@culture.gouv.fr 